\section{MEHR INFARKTE BEI ARBEITSLOSEN \\ Der Jobverlust geht auf's Herz}

Der Verlust des Arbeitsplatzes setzt dem Herzen ähnlich zu wie Rauchen, Diabetes oder Hochdruck. Das zeigten amerikanische Wissenschaftler in einer prospektiven Kohortenstudie mit 13451 Personen zwischen 51 und 75 Jahren. Die Probanden waren von 1992 bis 2010 alle zwei Jahre zu Berufstätigkeit und Gesundheit befragt worden. Während der Beobachtungszeit ereigneten sich 1061 Myokardinfarkte. Arbeitslose hatten ein deutlich höheres Infarktrisiko als Menschen mit Job. Wurden in der multivariaten Analyse neben Alter, Geschlecht etc. auch gesundheitliche Aspekte wie Raucherstatus, BMI, Hypertonie u.a. einbezogen, lag das Herzinfarktrisiko der Arbeitslosen durchschnittlich um 35\% höher als bei den Beschäftigten. Je häufiger sich der "Jobverlust" wiederholte, desto mehr Schaden nahm das Myokard. Nach der vierten Entlassung kletterte das Risiko um $63 \%$.

Arch Intern Med 2012; online 19. November; doi:10.1001/2013.jamainternmed.447

\title{
KARDIALES RISIKO
}

\section{Arrhythmie durch Pneumonie}

$12 \%$ von 32687 Patienten in den USA, die wegen einer Pneumonie stationär behandelt werden mussten, entwickelten in den ersten 90 Tagen nach der stationären Aufnahme eine Arrhythmie. Die Mortalität betroffener Patienten betrug nach 30 Tagen $18,4 \%$ (gegenüber $13,1 \%$ bei Patienten mit normalem Herzrhythmus), nach 90 Tagen $31 \%$ (vs. 20,8\%). Höheres Alter, Herzinsuffi- zienz in der Vorgeschichte und eine in den ersten beiden Tagen im Krankenhaus notwendige Beatmungs- oder Vasopressorentherapie waren mit dem Auftreten von Arrhythmien assoziiert. Als präventiv erwies sich die Einnahme von Betablockern.

Am J Med 2012; online 21. November; doi: 10.1016/j. amjmed.2012.08.005

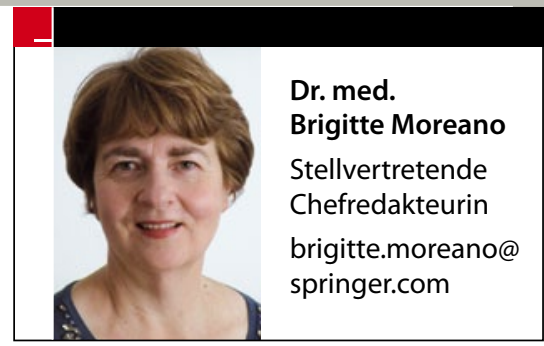

HOCHDRUCKTHERAPIE

\section{Mehr Hüftfrakturen in den ersten Wochen}

Wenn bei älteren Menschen eine medikamentöse Hochdrucktherapie eingeleitet wird, kann in den ersten Wochen ein erhöhtes Risiko für Hüftfrakturen bestehen, wie eine kanadische Studie belegt. In den ersten 45 Tagen einer medikamentösen Hochdrucktherapie erlitten alte Menschen 43\% mehr Hüftfrakturen als in den $45 \mathrm{Ta}$ gen zuvor und danach. Signifikant war die Risikosteigerung unter ACE-Hemmern (+ 53\%) und Betablockern (+58\%). Besonders gefährlich ist das Intervall zwischen Tag 15 und Tag 44.

Arch Intern Med 2012; online 19. November doi:10.1001/2013.jamainternmed.469

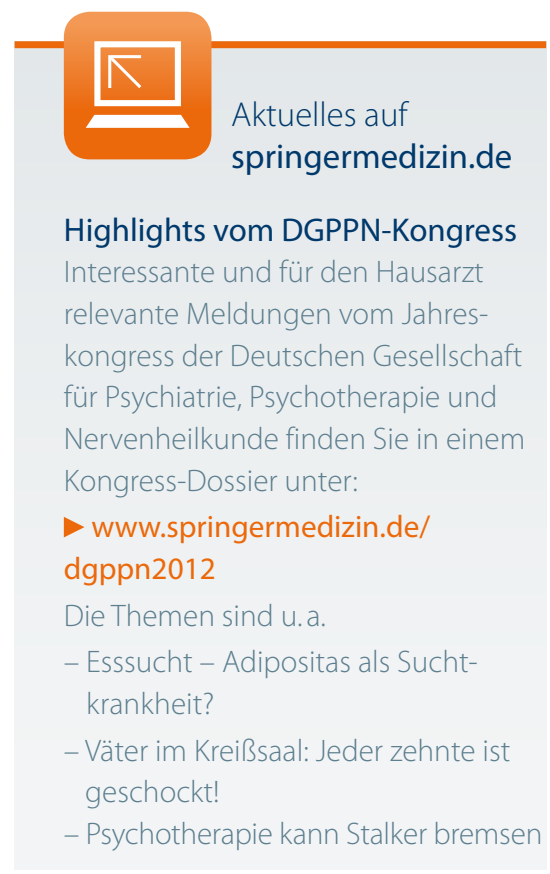

\section{POSTSTATIONÄRE KOMPLIKATIONEN}

\section{So kritisch ist die Zeit nach der Op.}

Die ersten Wochen nach der Klinikentlassung sind für frisch operierte Patienten riskant. In dieser Zeit ereigneten sich einer US-amerikanischen Studie zufolge über $40 \%$ aller Komplikationen. Wundinfektionen waren dabei am häufigsten.

Wissenschaftler der Stanford-Universität haben die Daten von 550000 Patienten nach einem chirurgischen Eingriff ausgewertet. In 17\% der Fälle traten Komplikationen auf. $42 \%$ ereigneten sich in den ersten 30 Tagen nach der Entlassung aus dem Krankenhaus. Am riskantesten war die Proktektomie (postoperative Komplikati- onsrate 15\%), gefolgt von Darmfistelchirurgie (13\%) und Pankreas-Operationen (11\%). Die Eingriffe, bei denen die meisten Komplikationen erst nach der Entlassung auftraten, waren Operationen an der Brust, bariatrische Chirurgie und nach BauchhernienOperationen mit 79\%, 69\% und $62 \%$ aller Komplikationen. Die Wahrscheinlichkeit, innerhalb von 30 Tagen erneut operiert werden zu müssen, war bei Patienten mit Komplikationen mehr als dreifach erhöht; ebenso das Risiko, in dieser Zeit zu versterben. 